\title{
ON SOME PROPERTIES OF GROUP RINGS
}

\author{
G. KARPILOVSKY
}

(Received 7 August; revised 11 December 1979)

Communicated by $\mathbf{H}$. Lausch

\begin{abstract}
Let Out $(R G)$ be the set of all outer $R$-automorphisms of a group ring $R G$ of arbitrary group $G$ over a commutative ring $R$ with 1 . It is proved that there is a bijective correspondence between the set Out $(R G)$ and a set consisting of $R(G \times G)$-isomorphism classes of $R$-free $R(G \times G)$-modules of a certain type. For the case when $G$ is finite and $R$ is the ring of algebraic integers of an algebraic number field the above result implies that there are only finitely many conjugacy classes of group bases in $R G$. A generalization of a result due to $\mathbf{R}$. Sandling is also provided.
\end{abstract}

1980 Mathematics subject classification (Amer. Math. Soc.): primary $20 \mathrm{C} 07$; secondary $20 \mathrm{C} 05$.

For $R$ a commutative ring and $A$ an $R$-algebra, let $\operatorname{Pic}_{R}(A)$ be the group of isomorphism classes of invertable $(A, A)$-bimodules for which the left and the right $R$-module structure coincide. The significance of $\operatorname{Pic}_{R}(A)$ in its relation to the automorphism group $\operatorname{Aut}_{R}(A)$ of the algebra $A$ was vividly demonstrated in Fröhlich (1973) which contained among other results the existence of a homomorphism $\Omega$ of $\operatorname{Aut}_{R}(A)$ into $\operatorname{Pic}_{R}(A)$ whose kernel is the group of all inner automorphisms of $A$.

In the first part of the paper we give an explicit description of $\Omega$ for the case when $A$ is a group algebra $R G$ of an arbitrary group $G$ over a commutative ring $R$ with 1 .

We shall say that two group bases $G_{1}$ and $G_{2}$ of $R G$ are conjugate in $R G$ if $u^{-1} G_{1} u=G_{2}$ for some invertible element $u$ in $R G$. It is a consequence of the above description that if $G$ is finite and if $R$ is the ring of algebraic integers of an algebraic number field then there are only finitely many conjugacy classes of group bases in $R G$. As another application of the above description we shall establish a criterion for when two isomorphic group bases in the integral group ring $Z G$ of a finite group $G$ are conjugate in $Q G$. In the second part of the paper we prove that if $G$ is an arbitrary 
group, $K$ is an arbitrary associative ring with $1, I(G)$ is the augmentation ideal of $K G$ and $N / M$ is an abelian section of $G$ then there is a $K$-module isomorphism

$$
\frac{I(N) K G}{I(N) I(G)+I(M) K G} \simeq K \otimes_{\mathrm{z}} N / M .
$$

Moreover, if $M / N$ is a normal abelian section then the above isomorphism is a $K G$ isomorphism where the $K G$-module structure on $K \otimes_{z} N / M$ is defined by

$$
g\left(k \otimes_{z} n M\right)=k \otimes_{z} g n g^{-1} \mathrm{M}(k \in K, n \in N, g \in G) .
$$

This result was established by R. Sandling in Sandling (1972) for the case $K=Z$.

\section{Automorphisms of $R G$ and $R(G \times G)$-modules}

Let $R G$ be a group algebra of an arbitrary group $G$ over a commutative ring $R$ with 1 . Denote by $U(R G)$ the group of units of $R G$ and by Aut $(R G)$ the group of all $R$-automorphisms of $R G$. For each $u \in U(R G)$ let $i_{u}$ be the inner automorphism of $R G$ defined by $i_{u}(x)=u^{-1} x u, x \in R G$. The group of inner automorphisms of $R G$ is defined as $\operatorname{In}(R G)=\left\{i_{u} \mid u \in U(R G)\right\}$. It is clear that $\operatorname{In}(R G)$ is a normal subgroup of Aut $(R G)$. We set Out $(R G)=$ Aut $(R G) / \ln (R G)$, the outer automorphism group of $R G$. We shall also write $R G=R H$ when $H$ is a group basis of $R G$.

We first need the following.

Lemma. Let $\bar{G}=G \times G$ and let for any $f \in \operatorname{Aut}(R G), M_{f}$ be the additive group of $R G$. Then $M_{f}$ is a (left) $R$-free $R \bar{G}$-module under the following action of $R \bar{G}$ :

$$
\begin{gathered}
\text { for each } t=\sum_{i=1}^{n} \alpha_{i}\left(a_{i}, b_{i}\right) \in R \bar{G} \text { and for each } x \in M_{f}, \\
t \circ x=\sum_{i=1}^{n} \alpha_{i} a_{i} x f\left(b_{i}^{-1}\right) .
\end{gathered}
$$

Proof. To prove that $M_{f}$ is an $R \bar{G}$-module it is enough to check that $M_{f}$ is a $\bar{G}$ module under the composition $(a, b) \circ x=a x f\left(b^{-1}\right),(a, b) \in \bar{G}, x \in M_{f}$. It is clear that for any $x_{1}, x_{2} \in M_{f},(a, b) \circ\left(x_{1}+x_{2}\right)=(a, b) \circ x_{1}+(a, b) \circ x_{2}$ and that $(1,1) \circ x=x$ for any $x \in M_{f}$. Let $(c, d) \in G$. Then for any $x \in M_{f}$,

$$
[(a, b)(c, d)] \circ x=a c x f\left(d^{-1}\right) f\left(b^{-1}\right)=(a, b) \circ\left[c x f\left(d^{-1}\right)\right]=(a, b) \circ[(c, d) \circ x] .
$$

Hence $M_{f}$ is an $R \bar{G}$-module. Since the group algebra $R G$ is an $R$-free module and since $r(1,1) \circ x=r x$ for any $r \in R, x \in R G$ it follows that $M_{f}$ is also $R$-free, regarded as $R \bar{G}$-module, proving the lemma. 
Denote by $P(R G)$ the set, consisting of all $R \bar{G}$-isomorphism classes $\left(M_{f}\right)$ of $R \bar{G}$ modules $M_{f}$, where $f$ ranges all elements of $\operatorname{Aut}(R G)$. Let also $M_{f}=M$ for $f=$ identity automorphism. We are now ready to prove the following.

THEOREM 1. The mapping $\Omega$ : Out $(R G) \rightarrow P(R G)$ defined by $\Omega[f \operatorname{In}(R G)]=\left(M_{f}\right)$ is a bijection. In particular, $f \in \operatorname{In}(R G)$ if and only if the $R \bar{G}$-modules $M$ and $M_{f}$ are isomorphic.

Proof. Let $\varphi \in \operatorname{Aut}(R G), u \in U(R G)$ and let $\psi=\varphi . i_{u}$. Consider the mapping :

$$
\mu: M_{\varphi} \rightarrow M_{\psi} \text { defined by } \mu(x)=x \varphi(u)
$$

for any $x \in M_{\varphi}$. It is clear that $\mu$ is an $R$-isomorphism of $R \bar{G}$-modules $M_{\varphi}$ and $M_{\psi}$. On the other hand, for any $(a, b) \in \bar{G}$ and for any $x \in M_{\varphi}$ we have

$$
\begin{aligned}
\mu((a, b) \circ x)=\mu\left(a x \varphi\left(b^{-1}\right)\right)=\operatorname{ax} \varphi\left(b^{-1}\right) \varphi(u) & =a x \varphi(u) \varphi\left(u^{-1} b^{-1} u\right)=a \mu(x) \psi\left(b^{-1}\right) \\
& =(a, b) \circ \mu(x) .
\end{aligned}
$$

Hence $\mu$ is an $R \bar{G}$-isomorphism and therefore the map $\Omega$ is well defined. Now let

$$
\theta: M_{\Phi} \rightarrow M_{f} \text { be an } R \bar{G} \text {-isomorphism and let } u=\theta(1) \text {. }
$$

Then for any $(a, b) \in \bar{G}$ and for any $x \in M_{\varphi}$, the equality $\theta[(a, b) \circ x]=(a, b) \circ \theta(x)$ implies

$$
\theta\left(\operatorname{ax} \varphi\left(b^{-1}\right)\right)=a \theta(x) f\left(b^{-1}\right) .
$$

Taking $x=b=1$ in (1) we obtain $\theta(a)=a u$ for any $a \in G$ and since $\theta$ is necessarily an $R$-isomorphism of $R \bar{G}$-modules $M_{\varphi}$ and $M_{f}$ it follows that

$$
\theta(x)=x u \text { for any } x \in M_{\varphi} .
$$

Next choose $a=x=1$ in (1), whence

$$
\theta\left[\varphi\left(b^{-1}\right)\right]=u f\left(b^{-1}\right)
$$

for any $b \in G$ and it follows from (2) that $\varphi(g) u=u f(g)$ for any $g \in G$. Hence

$$
\varphi(x) u=u f(x) \text { for any } x \in M_{\varphi}
$$

Therefore $R G u=u R G$ and it follows from (2) that $R G=R G u$ and $R G=u R G$ whence $u \in U(R G)$. It follows from (3) that for any $x \in M_{\varphi}$,

$$
f(x)=u^{-1} \varphi(x) u, \text { that is, } f=i_{u} \varphi .
$$

This shows that the map $\Omega$ is one-to-one and since $\Omega$ is obviously surjective, the proof is complete. 
Until now $G$ could have been any group. The assumption that $G$ is finite will now be brought into play. Let $Z G=Z H$ where $G$ is a finite group, $G \cong H$ and let $H$ be a normalized group basis of $Z G$, that is $H$ is a basis consisting of units having augumentation 1. It is natural to ask whether there is a unit $u$ in $Z G$ such that $H=u^{-1} G u$. That this is not always the case was first proved in 1966 by $\mathrm{S}$. D. Berman and A. R. Rossa (Berman and Rossa (1966)). Therefore we are led to ask whether for an arbitrary finite group $G$ the number of conjugacy classes of group bases in $Z G$ is finite. For the case $R=Z$ the following corollary gives a positive answer to this question.

COROLLARY 1. There are only finitely many conjugacy classes of group bases in RG where $R$ is the ring of algebraic integers of an algebraic number field and $G$ is a finite group.

ProOF. The application of Zassenhau's Theorem (Curtis and Reiner (1962)) and of the above Theorem implies that the group $\operatorname{Aut}(R G) / \operatorname{In}(R G)$ is finite. Let

$$
\operatorname{Aut}(R G)=\operatorname{In}(R G)+\operatorname{In}(R G) \varphi_{2}+\ldots+\operatorname{In}(R G) \varphi_{t}
$$

be the coset decomposition of Aut $(R G)$ with respect to $\operatorname{In}(R G)$. Suppose that $H$ is an arbitrary group basis of $R G$. Since $|H|=|G|$ there exists only a finite number of nonisomorphic group bases in $R G$, say, $G_{1}, G_{2}, \ldots, G_{n}$. Hence $H \cong G_{i}$ for some $i \in\{1,2, \ldots, n\}$ and therefore there exists $f \in \operatorname{Aut}(R G)$ such that $f\left(G_{i}\right)=H$. Since $f=\theta \varphi_{j}$ for some $\theta \in \operatorname{In}(R G)$ and some $j \in\{1,2, \ldots, t\}$ then $f\left(G_{i}\right)=u^{-1} \varphi_{j}\left(G_{i}\right) u$ for some $u \in U(R G)$, that is $H$ is conjugate to $\varphi_{j}\left(G_{i}\right)$, proving the result.

Another consequence of Theorem 1 is the following.

COROLLARY 2. Let $Z G=Z H$ where $G$ is finite, $H \cong G$ and let fbe the automorphism of the rational group algebra $Q G$ which is the extension of the isomorphism $H \rightarrow G$ by $Q$-linearity. Denote by $M$ (respectively $\left.M_{f}\right)$ the $Q(G \times G)$ module $Q G$ defined by $(a, b) \circ x=a x b^{-1},(a, b) \in G \times G, x \in Q G$ (respectively the $Q(G \times G)$-module $Q G$ defined $\left.b y(a, b) \circ x=\operatorname{axf}\left(b^{-1}\right)\right)$. Then $H$ is conjugate to $G$ in $Q G$ if and only if $\chi=\chi_{f}$ where $\chi$ (respectively $\chi_{f}$ ) is the character of $G \times G$ afforded by $M$ (respectively $M_{f}$ ).

Proof. All we have to do is to notice that $Q(G \times G)$-modules $M$ and $M_{f}$ are isomorphic if and only if $\chi=\chi_{f}$ and apply Theorem 1 .

We now digress for a moment to make a few remarks. Note that the character table of a finite group $G$ is determined by $Z G$ (Saksonov (1966)). Since $S_{n}$ is determined by its character table (Nagao (1957)) it follows that $S_{n}$ is determined up to isomorphism by $Z S_{n}$. Hence the application of a result due to G. Peterson 
(Peterson (1976)) implies that in $Q S_{n}, S_{n}$ is conjugate to any normalized group basis in $Z S_{n}$. It is not however known whether any normalized group basis in $Z S_{n}$ is conjugate in $Z S_{n}$ to $S_{n}$. That any normalized group basis of $Z S_{3}$ is conjugate in $Z S_{3}$ to $S_{3}$ is a result due to Hughes and Pearson (Hughes and Pearson (1972)). Finally, note that there is an intimate connection between the conjugacy of group bases and isomorphism problem. Indeed as it was pointed out in Whitcomb (1968) that if $G$ is a $p$-group of class 2 and if every normalised group bases in $Z G$ is conjugate in $O_{p} G$ to $G$ where $O_{p}$ the ring of $p$-adic integers, then any $p$-group of class $\leqslant 5$ is determined by its integral group ring.

\section{Module Isomorphisms}

In this section $G$ always denotes an arbitrary group and $K$ denotes an associative ring with 1 . The augmentation ideal $I(G)$ of the group ring $K G$ is the kernel of the homomorphism from the group ring $K G$ to $K$ induced by collapsing $G$ to the unit group. If $C$ and $D$ are subsets of $K G$, define the Lie bracket $(C, D)$ as the subgroup of the additive group of $K G$ generated by all $(c, d)=c d-d c, c$ in $C, d$ in $D$. Let $N$ and $M$ be subgroups of $G$. Then the identity $(a-1, b-1)=b a\left(a^{-1} b^{-1} a b-1\right), a, b \in G$ implies $K G . I([N, M]) \leqslant K G(I(N), I(M))$ and in particular

$$
K G . I([N, G]) \leqslant K G(I(N), I(G)) .
$$

The homomorphism $G \rightarrow K \otimes_{Z} G / G^{\prime}$ determined by $g \rightarrow 1 \otimes g G^{\prime}$ is called the universal homomorphism of $G$ into the additive group of a $K$-module. We shall denote the kernel of this homomorphism by $G^{\langle K\rangle}$.

In this section we shall prove the following result.

THEOREM 2. Let $N / M$ be an abelian section of $G$. Then there is a K-module isomorphism

$$
\frac{I(N) K G}{I(N) I(G)+I(M) K \bar{G}} \cong K \otimes_{\mathrm{z}} N / M .
$$

Moreover if $M / N$ is a normal abelian section then the above isomorphism is a $K G$ isomorphism, where the $K G$-module structure on $K \otimes_{Z} N / M$ is defined by $g(k \otimes n M)=k \otimes g n g^{-1} M(g \in G, k \in K, n \in N)$.

Proof. Let $J=I(N) I(G)+I(M) K G, L=I(N) K G$ and let $T$ be a right transversal of $G$ relative to $N$ containing 1 . We first observe that $L$ is a free $K$-module on the basis $\{(n-1) t \mid t \in T, 1 \neq n \in N\}$ and that

$$
\Psi: L / J \rightarrow K \otimes_{Z} N / M
$$

where $\Psi[(n-1) t+J]=1 \otimes n M$ is a $K$-module epimorphism. 
The mapping

$$
\varphi: K \otimes_{Z} N / M \rightarrow L / J
$$

defined by $\varphi(1 \otimes n M)=(n-1)+J$ is a $K$-module homomorphism. It is easy to see that $\varphi$ is the inverse of $\psi$, proving the first part of the theorem. Suppose that $N / M$ is a normal abelian section. Since the set $X=\{(n-1)+J \mid n \in N\}$ is a generating set of a $K$-module $L / J$ the second part of the theorem will be established once we verify that for any $g \in G$ and any $n \in N, \Psi[g(n-1)+J]=g \Psi[(n-1)+J]$. Since the last equality is a consequence of the identity $g(n-1)=\left(g n g^{-1}-1\right) g$ and the congruence $\left(g n g^{-1}-1\right) g \equiv g n g^{-1}-1(\bmod J)$, the result follows.

The following corollaries are well known for the case $K=Z$ (see Sehgal (1978)).

COROLlary 1. Let $N$ be a subgroup of $G$. Then there is a K-module isomorphism

$$
\frac{I(N) K G}{I(N) \cdot I(G)} \cong K \otimes_{Z} N / N^{\prime} .
$$

Moreover, if $N \triangleleft G$ then the above isomorphism is a $K G$-isomorphism where the $K G$ module structure on $K \otimes_{\mathrm{Z}} N / N^{\prime}$ is defined by $g\left(k \otimes{ }_{n} N^{\prime}\right)=k \otimes g n g^{-1} N^{\prime}$ $(g \in G, k \in K, n \in N)$.

Proof. Since $I\left(N^{\prime}\right) K G \subseteq I(N)^{2} K G \subseteq I(N) . I(G)$ the application of Theorem 2 for the case $M=N^{\prime}$ implies the desired isomorphism.

COROLlaRY 2. Let $N$ be a normal subgroup of $G$. Then there is a KG-isomorphism

$$
\frac{I(N) K G}{I(N) . I(G)+I(G) . I(N)} \cong K \otimes_{z} N /[N, G]
$$

where the $K G$-module structure on $K \otimes_{Z} N /[N, G]$ is defined by

$$
g(k \otimes n[N, G])=k \otimes\left(g n g^{-1}\right)[N, G] \quad(k \in K, n \in N, g \in G)
$$

Proof. If $M=[N, G]$ then $N / M$ is a normal abelian section of $G$ and all we have to do is to prove that

$$
I(N) I(G)+I(G) \cdot I(N)=I(N) . I(G)+I(M) K G .
$$

It follows from (4) that

$$
I(M) K G \subseteq K G(I(N), \quad I(G)) \subseteq I(N) I(G)+I(G) . I(N),
$$

whence

$$
I(N) . I(G)+I(M) K G \subseteq I(N) . I(G)+I(G) I(N)
$$


On the other hand, the identity

$$
\begin{aligned}
(g-1)(n-1)=( & -1)(g-1)+(n-1)(g-1)\left(g^{-1} n^{-1} g n-1\right) \\
& +(g-1)\left(g^{-1} n^{-1} g n-1\right)+\left(g^{-1} n^{-1} g n-1\right) \\
& +(n-1)\left(g^{-1} n^{-1} g n-1\right)
\end{aligned}
$$

implies

$$
I(G) . I(N) \subseteq I(N) . I(G)+I(M) K G .
$$

Hence

$$
I(N) . I(G)+I(G) . I(N) \subseteq I(N) I(G)+I(M) K G,
$$

proving (5) and thus completing the proof.

The next corollary is known for the case when $K$ is a commutative ring with 1 (see Bergman and Dicks (1975)).

Corollary 3.

$$
G \cap\left(1+I(G)^{2}\right)=G^{\langle K\rangle} .
$$

Proof. Let $\delta: G \rightarrow K \otimes G / G^{\prime}$ where $\delta(g)=1 \otimes g G^{\prime}$. Then $\delta$ determines a $K$ module homomorphism $\mu: I(G) \rightarrow K \otimes_{Z} G / G^{\prime}$ where $\mu(g-1)=1 \otimes g G^{\prime}$. Since $\delta(g)=\mu(g-1)$, so $g \in G^{\langle K\rangle}$ if and only if $g-1 \in \operatorname{Ker} \mu$. By taking the case $N=G$ and $M=G^{\prime}$ in the proof of Theorem 2, we see that $\operatorname{Ker} \mu=I(G)^{2}$, as desired.

\section{Acknowledgement}

The author wishes to thank the referee for his valuable remarks.

\section{References}

G. M. Bergman and W. Dicks (1975), 'On universal derivations', J. Algebra 36, 193-211.

S. D. Berman and A. R. Rossa (1966), 'Integral group rings of finite and periodic groups', Algebra and Math. Logic, Izdat Kiev, Univ. Kiev, pp. 44-53.

C. W. Curtis and I. Reiner (1962), Representation theory of finite groups and associative algebras (Interscience, New York and London).

A. Fröhlich (1973), 'The Picard group of noncommutative rings, in particular of orders', Trans. Am. Math. Soc. 180, 1-46.

I. Hughes and K. R. Pearson (1972), 'The group of units of the integral group ring $Z S_{3}{ }^{\prime}$ Canad. Math. Bull. $15,529-534$.

H. Nagao (1957), 'On the groups with the same table of characters as symmetric groups' J. Inst. Polytech. Osaka City Univ. (Ser. A8), 1-8. 
G. Peterson (1976), 'Automorphisms of the integral group ring of $S_{n}$ ', Proc. Amer. Math. Soc. 59, 14-18. A. I. Saksonov (1966), 'Certain integer valued rings associated with a finite group', Dokl. Akad. Nauk SSSR 171, 529-532.

R. Sandling (1972), 'Note on the integral group ring problem', Math. Z. 124, 255-258.

S. K. Sehgal (1978), Topics in group rings (Marcel Dekker, Inc., New York and Basel).

A. Whitcomb (1968), The group ring problem ( $\mathrm{PhD}$ thesis, University of Chicago).

Department of Mathematics

La Trobe University

Bundoora, Victoria, 3083

Australia 